\title{
3 Research Square

\section{Clinical Efficacy of Endoscopic Technique in the Treatment of Gastrointestinal Anastomotic Complications: a Retrospective Study}

Shenghe Deng

Huazhong University of Science and Technology

Junnan Gu

Huazhong University of Science and Technology

Yinghao Cao

Huazhong University of Science and Technology

Fuwei Mao

Huazhong University of Science and Technology

Ke Liu

Huazhong University of Science and Technology

Jiliang Wang

Huazhong University of Science and Technology

Ke Wu

Huazhong University of Science and Technology

Kailin Cai ( $\nabla$ caikailin@hust.edu.cn )

Huazhong University of Science and Technology

\section{Research Article}

Keywords: Endoscopy, Anastomotic complications, Anastomotic stenosis, Anastomotic obstruction, Anastomotic fistula

Posted Date: September 9th, 2021

DOl: https://doi.org/10.21203/rs.3.rs-864798/v1

License: (c) (i) This work is licensed under a Creative Commons Attribution 4.0 International License. Read Full License 


\section{Abstract}

Background: To evaluate the safety and effectiveness of endoscopic technique in treating postoperative anastomotic complications of digestive tract.

Methods: Clinical data of patients received endoscopic treatment in our hospital due to anastomotic complications after gastrointestinal surgery from January 2015 to December 2018 were collected for retrospective analysis. Endoscopic intervention was used for postoperative anastomotic complications in all the included cases. The time of the intervention measures, laboratory examination, incidence of complications and postoperative follow-up were observed and analyzed.

Results: A total of 88 patients were included in the study, including 43 patients with anastomotic stenosis,22 with anastomotic obstruction,23 with anastomotic fistula. For anastomotic obstruction patients, 36 patients with anastomosis were successfully treated with endoscopy. For anastomotic obstruction patients, 18 cases were successfully treated with endoscopy. For anastomotic fistula patients, 21 cases were successfully treated with endoscopy.

During follow-up after the endoscopic procedure, 4 patients with anastomotic stenosis needed endoscopic intervention again, 3 cases undertook surgical intervention and 2 cases maintained acceptable defecation function by intermittent dilation with a plastic dilater. one patients with anastomotic obstruction had stent displacement and was removed. For the anastomotic fistula patients,one case relapsed and then underwent endoscopic intervention again, three cases converted to surgical intervention. Preoperative and postoperative blood biochemical examination had no significant statistical significance

Conclusion: Endoscopic treatment of anastomosis complications was effective for some of the patients, especially for those with anastomosis stenosis or stricture, considering its saftey, it might be the first choice for anastomosis complications.

\section{Introduction}

In the past few decades, gastrointestinal malignancies have become one of the five most common malignancies with high morbidity and mortality. The conventional treatment of gastrointestinal malignant tumors is mainly surgery, supplemented by radiotherapy and chemotherapy. With the deepening of research progress, comprehensive tumor treatment has made great progress in patients with early malignant tumors; at the same time, with the improvement of health care, the mortality rate of malignant tumors has declined[1]. Surgical resection is still the preferred treatment for resectable tumors of the digestive tract. As digestive tract surgery involves reconstruction of the digestive tract, postoperative anastomotic complications are easy to occur. Anastomotic complications are one of the most common and dangerous complications after digestive tract anastomosis, characterized by high incidence and high mortality, which can increase the risk of postoperative recurrence and negatively affect the long-term prognosis of patients[2, 3]. 
The common anastomotic complications after gastrointestinal surgery include: anastomotic bleeding, anastomotic fistula/leakage (AF/AL), anastomotic obstruction (inflammatory obstruction, input/output loop obstruction), anastomotic stricture (AS). At present, the risk factors and pathophysiological mechanism of postoperative anastomotic complications of digestive tract have not been fully defined. The main causes may be related to nutrition, anastomotic technology, anastomotic tension, anastomotic mode, bilateral blood circulation, digestive tract obstruction, infection, obesity, diabetes, preoperative and postoperative radiotherapy and chemotherapy, perioperative preparation and other factors[4]. For the management of postoperative complications of anastomosis, the common treatment methods include conservative treatment, endoscopic treatment and surgical treatment. Conservative treatment mainly includes anti-infection, fasting and symptomatic support. For patients with severe symptoms, most clinicians still consider surgical treatment. With the maturity of endoscopic techniques and the development of endoscopic surgery, endoscopic techniques are more and more widely used to treat postoperative anastomotic complications. Endoscopic techniques in the treatment of anastomotic leakage mainly include endoscopic jejunal nutrition tube placement, metal clip closure, stent placement, tissue sealants, suturing, vacuum-assisted closure therapy[5]; Endoscopic treatment of complications of anastomotic stenosis mainly includes placement of temporary self-expandable metal stent (SEMS), endoscopic incision (EI), balloon dilatation (EBD)[6]; In the treatment of anastomotic obstruction (AO), different therapeutic schemes are selected according to different types of obstruction, mainly including endoscopic jejunal nutrition tube placement, metal stent placement, endoscopic drainage[7].

Although endoscopic techniques have been supported by most people in the treatment of postoperative complications of digestive tract, there are few reports on the safety of endoscopic intervention. The data of patients with endoscopic anastomotic complications treated by our medical team were analyzed to investigate the safety and efficacy of endoscopic treatment of postoperative anastomotic complications of digestive tract retrospectively .

\section{Methods}

\section{Study design}

From January 2015 to December 2018, the clinical data of all patients who had anastomotic complications during digestive tract operation in Gastrointestinal Surgery of Wuhan Union Hospital and who were referred from other medical institutions to deal with anastomotic complications and these received endoscopic intervention were analyzed retrospectively. This study was a single-center retrospective study.

\section{Outcome measures}

All patients who were treated with endoscopy for anastomotic complications after digestive tract operation were enrolled in this study. The time of occurrence, intervention measures, technical success rate, changes of laboratory examination indicators before and after operation,treatment success rate, complications, intervention times and postoperative follow-up were analyzed for safety and 
effectiveness. Regular follow-up were conducted to all enrolled patients after discharge by telephone, inhospital examination, outpatient follow-up.

\section{Satistical analysis}

Spss23.0 software was used for statistical analysis of all data. Fisher's test or chi-square test were used to evaluate the proportion difference between groups. Continuous variables were expressed as mean \pm standard deviation, and compared by t-test, $\mathrm{P}<0.05$ was considered significant.

\section{Ethics statement}

Informed consent was obtained from patients prior to participation in the study. Work on human complied with the principles set out in the "Helsinki Declaration "[8].The study protocol was approved by the ethics committee and was registered in the Chinese Clinical Trial Registry[No.:ChiCTR1900023804].

This study was reviewed by the Ethics Committee of Tongji Medical College, Huazhong University of Science and Technology (No. 2018-S377, dated 11 July 2018). All procedures performed in this study involving human participants were in accordance with the 1964 Declaration of Helsinki and its later amendments or comparable ethical standards. Informed consent was submitted by all subjects when they were enrolled. This study was registered in the Chinese Clinical Trial

Registry[No.:ChicTR1900023804].

\section{Results}

\section{Clinicopathological characteristics of patients with different types of anastomotic complications}

All the 88 patients were treated under endoscopy, and no related adverse events occurred during the operation. Among the enrolled patients, there were 43 cases of anastomotic stenosis of digestive tract, including 20 cases of upper and lower gastrointestinal stenosis and 23 cases of upper and lower gastrointestinal stenosis respectively. In the upper digestive tract anastomotic stenosis, there were 12 male patients and 6 female patients, with an average symptom onset time of $3.81 \pm 3.46$ months, 16 benign stenosis cases and 4 malignant stenosis cases. Preoperative Stooler grade[9]: 3 cases of grade II, 10 cases of grade III, and 7 cases of grade IV.The average length of hospitalization was $4.94 \pm 2.08$ days. The average postoperative feeding time was $3.1 \pm 5.0$ days. In the lower digestive tract anastomotic stenosis, the symptom onset time of the patients was $7.97 \pm 8.69$ months, including 21 cases of benign stenosis and 2 cases of malignant stenosis. Preoperative Truong anastomotic stenosis classification[10]: 2 cases of grade I, 12 cases of grade II and 9 cases of grade III.The stenosis length was $2.3 \pm 1.36 \mathrm{~cm}$, the average length of hospitalization was $5.86 \pm 4.02$ days, and the postoperative feeding time was $3.0 \pm 1.97$ days. The average feeding time after operation for all patients with gastrointestinal stenosis was $3.38 \pm$ 3.0 days.(Fig. $1 \mathrm{~A}, 1 \mathrm{C}$,Table $\mathrm{s} 1$ ).

There were 22 cases of anastomotic obstruction of digestive tract, all of which were upper gastrointestinal tract. There were 15 cases of inflammatory obstruction and 7 cases of input/output loop 
obstruction. The onset time of symptoms was $12.5 \pm 4.49$ days. Postoperative feeding time was $8.65 \pm$ 3.44 days, mainly nasogastric feeding through jejunal nutrient canal, and the average length of hospital stay was $26.13 \pm 14.72$ days.(Fig. 1 G,Table s2).

There were 23 cases of anastomotic fistula, and the onset time of symptoms was $12 \pm 6.28$ days. The average postoperative feeding time was $5.44 \pm 2.84$ days, and nasal feeding was dominant. The average length of hospitalization was $26.21 \pm 14.50$ days. Low DE et al[11] anastomotic classification: 8 cases of grade A, 12 cases of grade B, 3 cases of grade C.(Fig. 2A-2B,Table s3).

\section{Endoscopic intervention measures and management of anastomotic complications}

Among the endoscopic interventions for upper gastrointestinal anastomotic stenosis, there were 5 cases of upper gastrointestinal stent implantation, 5 cases of endoscopic incision, 4 cases of balloon dilatation, 1 case of endoscopic gastrostomy, 4 cases of surgical treatment and 1 case of conservative treatment after failure of intraoperative endoscopic treatment. Short-term postoperative complications: 1 patient with stent placement had stent displacement 20 days after the operation.

Endoscopic intervention measures for lower gastrointestinal anastomotic stenosis: stent implantation in 12 cases, endoscopic incision in 4 cases, balloon dilatation in 3 cases, stenosis incision plus stent implantation in 3 cases, and surgical treatment in 1 case after intraoperative endoscopy.Short-term postoperative complications occurred in 4 patients: 1 patient underwent stent displacement on the 4th day after surgery;Blood stool occurred 10 days after stent implantation in 1 case. One patient with subcutaneous emphysema, fever and suspected microperforation underwent conservative treatment after incision and stent implantation. Postoperative abdominal pain and discomfort occurred repeatedly in 1 patient with stent implantation (Fig. 1B,1D,Table s1).

Digestive tract obstruction: Among the patients with inflammatory obstruction, 15 cases were successfully placed with jejunal nutrition tube under endoscope, and 1 case received surgical treatment with no remission of postoperative symptoms. In input/output loop obstruction, 4 cases underwent successful endoscopic treatment, including 2 cases of input loop drainage under endoscopy and 2 cases of output loop obstruction with endoscope stent placement, three patients were treated by surgery after failure of intraoperative endoscopic treatment.(Fig. 1H,Table s2)

In the endoscopic intervention of anastomotic fistula of digestive tract, 8 cases were placed with jejunal nutrition tube under endoscope, 1 case was placed with stent, and 7 cases were clipped with metal clip. Among them, 5 cases of esophageal fistula were clipped with over-the-scope Clip(OTSC), and 2 cases with small esophagojejunostomy fistula were clipped with metal Clip, One case of endoscopic examination suspected anastomotic ischemia received surgical treatment and jejunal nutrition tube implantation.Short-term postoperative complications: 1 case of esophageal fistula developed high fever after OTSC clipping, endoscopic examination revealed that endoscopic jejunal nutrition tube placement was performed after failure of clipping. 
In the lower digestive tract anastomotic fistula, 4 cases were placed with full-coverd metal stent, among which 1 case was found to have a small fistula closed with metal clamp 15 days after the stent was taken. Two patients with rectovaginal fistula: 1 case treated with full coverd metal stent and vacuumassisted closure therapy, no significant relief was found and surgical treatment was selected,anothor patient was treated with vacuum-assisted closure therapy(Fig. 2C-2D,Table s3).

\section{Changes in laboratory examination after endoscopic intervention}

After 3 days of endoscopic treatment for anastomotic stenosis, anastomotic fistula and anastomotic obstruction, the ratio of white blood cells and neutrophils of the patients after the operation did not increase significantly compared with that before the operation, and there was no significant statistical significance.(Fig. 3,Table s7)

\section{Postoperative follow-up outcomes}

In the follow-up of patients with upper gastrointestinal anastomotic stenosis for 1 month after endoscopic therapy, 1 patient after stent implantation was found to still have stenosis and then underwent endoscopic balloon dilatation. Stooler classification: 10 cases of Grade 0, 2 cases of Grade I, 1 case of Grade II, and 2 cases of Grade III.During the 3-month follow-up, Stooler classification:11 cases of grade 0,3 cases of grade I, 1 case of Grade III. One patient was placed into the jejunal nutrition tube again due to recurrent acid reflux and abdominal pain. The results of 1-year follow-up showed that the patients died due to tumor metastasis after gastrostomy, and the symptoms of the remaining patients with upper gastrointestinal anastomotic stenosis were all relieved. In the follow-up of patients with lower gastrointestinal anastomotic stenosis 1 month after operation, postoperative Truong anastomotic stenosis grade: 13 cases of grade 0 -I, 5 cases of grade II, and 3 cases of grade III, among which 4 cases of stents were still not removed. Three patients with grade III stenosis received surgical treatment without significant relief. During the follow-up 3 months after surgery, postoperative Truong anastomotic stenosis was classified into 16 cases with grade $0-I$ and 2 cases with grade II. 1 patient after stent removal and was reinserted; 1 patient had abdominal pain and was found to have edema around the anastomotic site after reexamination, and underwent stent removaland radial incision.During the follow-up of 1 year after operation, 2 patients were lost to follow-up, the remaining patients' symptoms were relieved, and no additional auxiliary intervention was needed, and no recurrence was found.(Fig. 1E-1F,Table s4).

Among the patients with digestive tract anastomotic obstruction, 1 case of the patients after stent implantation had stent displacement and returned to hospital for stent removal, and the anastomotic site returned to normal.After 3 months to 1 year of follow-up, all patients were in remission and returned to normal diet and exhaust and defecation. Stents were removed in all patients, and no patients experienced recurrence.(Fig. 11,Table s5).

Among the patients with anastomotic fistula in the upper digestive tract, 5 patients with anastomotic fistula were followed-up 1 month after operation. The patients were followed-up 3 months after the operation, and the symptoms were all relieved. The patients were followed up 6 months after the 
operation, a patient was treated with OTSC for anastomotic fistula recurrence, and the anastomotic stoma was treated by OTSC again.After 1 year of follow-up, all patients with anastomotic fistula had been cured, without other anastomose-related complications. In patients with lower digestive tract anastomotic fistula: 1 month follow-up, 1 case selected surgical exploration, 1 case of rectovaginal fistula treated with vacuum-assisted closure therapy and anastomotic fistula was smaller than before. Six months vacuum-assisted closure therapy, rectovaginal fistula was treated by surgery. Results of 1year follow-up showed none of the patients died and the function of exhaust and defecation was normal. (Fig. 2E-2F,Table s6).

\section{Discussion}

In recent years, endoscopic therapy is the first-line treatment for many benign and malignant gastrointestinal anastomos-related complications. As a minimally invasive, effective and intuitive diagnosis and treatment method, endoscopic technology has become more and more popular among clinicians.

There are various endoscopic treatments for different anastomotic complications. The treatment principle of anastomotic stenosis is to relieve stenosis and restore intestinal patency. In the treatment of gastrointestinal anastomotic stenosis, common endoscopic techniques mainly include: SEMS placement, endoscopic stenosis incision and endoscopic balloon dilatation. Temporary SEMS implantation has become a treatment option for postoperative anastomotic complications of digestive tract, but the relatively high mobility rate and high cost of stents hinder the efficacy[12]. It has been reported that endoscopic stent placement is feasible, safe and has a high success rate in the treatment of anastomotic complications after upper gastrointestinal surgery, especially some stubborn anastomotic stenosis, and the advantages of treating anastomotic complications in high-risk patients with this minimally invasive method are obvious $[13,14]$. In our study, 43 patients with postoperative anastomotic stenosis were treated by endoscopy, including 17 cases of SEMS implantation, 2 cases of early postoperative complications with stent displacement. We can conclude that the covered stent has a significant effect in the treatment of anastomotic stenosis.

EBD is a widely used endoscopic technique at present, which has a high success rate in the treatment of postoperative anastomotic stenosis of digestive tract, with fewer complications and significant clinical effect[15].Araujo et al. believe that EBD is the best treatment method for patients with postoperative anastomotic stenosis of digestive tract, but multiple interventions are needed and recurrence should be paid attention[16]. Truong et al. and Fregonese et al. found in their study that the recurrence rate after endoscopic balloon dilatation was $6 \%$ and $20 \%$ respectively $[10,17]$. The reasons for recurrence after EBD are still unclear. In our study, 7 patients received EBD. During the follow-up, all patients were relieved of postoperative stenosis and obstruction. EBD can be considered as a non-invasive treatment for anastomotic stenosis, which is safe and effective, but may require multiple interventions to achieve the desired effect. 
In addition to endoscopic stent placement and balloon dilatation, $\mathrm{El}$ is also an important means to relieve anastomotic stenosis. However, this type of operation has many wounds, which can easily lead to perforation, bleeding, infection and other complications. Tan et al. considered El as a feasible, safe and effective method for the treatment of refractory anastomotic stenosis[18]. Osera et al. also concluded that $\mathrm{El}$ is a safe and effective method with low incidence of complications for poor expansion effect or refractory anastomotic stenosis[19]. In our previous research found that for patients with colorectal anastomotic stenosis, endoscopic radial anastomotic incision is a safe and effective treatment method[20]. We think that El is a safe and effective method, but skilled endoscopic surgeons are required to operate it to prevent the occurrence of serious complications such as perforation and bleeding caused by improper level and depth of incision. In our study,among the 43 patients, 4 patients had postoperative complications and no serious adverse events or death. During the 1-year follow-up, obstruction was successfully relieved in 33 patients. Our research data suggest that endoscopic technique is a safe and effective technique for the treatment of anastomotic stenosis.

$\mathrm{AF}$ is also one of the important factors affecting postoperative quality of life, increasing morbidity and mortality, increasing hospitalization expenses and long-term recurrence[21]. According to literature reports, the incidence of anastomotic fistula is $1-10 \%$ or even as high as $30 \%[22,23]$, and the total mortality of digestive tract anastomotic fistula is still between $10-25 \%$ even after early diagnosis and active treatment[24].Some studies believe that the treatment of anastomotic fistula by simply placing jejunal nutrition tube is not effective, but it can be prepared for the other treatmen[25]. We found that the placement of nutritional tubes for the treatment of anastomotic fistula was a safe and effective treatment plan. It is considered that endoscopic stent placement can effectively control the anastomotic complications, especially anastomotic leakage after gastrointestinal surgery. Julietta et al. also believed that endoscopic stent is an effective and minimally invasive treatment for anastomotic complications after upper gastrointestinal surgery[26]. Among the 23 patients with anastomotic fistula included in the group, 7 patients were treated with metal clamp for fistula closure,no postoperative complications occurred in all the patients. A multicenter retrospective study found that the long-term success rate of patients with acute perforation or anastomotic fistula treated with metal clips reached $90 \%$ and $73.3 \%$, respectively[27]. Endoscopic stent implantation plus endo-sponge low negative pressure continuous suction or endo-sponge low negative pressure continuous suction are also considered as a treatment method. Mussetto et al. believed that Endo-Spopnge treatment of colorectal anastomotic fistula was a successful and safe method for appropriate patients, with mild to moderate discomfort, but could shorten wound closure and length of hospital stay[28]. However, in the case of severe fistula edema or large fistula, it is difficult to accurately and effectively clamp the fistula. Among the 23 patients with anastomotic fistula, the average postoperative feeding time was $5.44 \pm 2.84$ days, and the average postoperative hospital stay was $26.21 \pm 14.50$ days. In conclusion, we believe that endoscopic technique is a safe, minimally invasive and effective technique for the treatment of AF.

Besides anastomosis stenosis, postoperative obstruction of the digestive tract may also be caused by inflammatory edema of the anastomosis, intestinal angulation deformity of the input/output loop. For this kind of special obstruction, the pathogenesis may be as follows: extensive adhesion and separation 
of abdominal tissues after digestive tract surgery, extensive exudation of abdominal cavity caused by normal tissue damage, intraperitoneal suture or foreign tissue. In the case of obstruction caused by inflammatory oedema at the anastomotic site,we believe that endoscopic nasojejunal nutrient catheter is a safe and effective treatment for inflammatory or dynamic obstruction. In this study, a total of 15 patients with inflammatory obstruction were placed with nasojejunal nutrition tube under endoscopy. With the help of nasojejunal nutrition tube drainage, nasal feeding, anti-infection and symptomatic support, 14 patients successfully cured inflammatory obstruction. 7 cases of anastomotic obstruction were anastomotic input/output loop obstruction, and there were few reports on the treatment of input loop and output loop obstruction, obstruction caused by anastomotic loop angle deformity after gastrectomy has become a difficult problem in clinical treatment. Traditional surgical operation is more traumatic to patients, and patients often refuse surgery due to poor physical condition and tolerance, rapid disease progression and high incidence of complications. Based on our clinical data, we found that the main causes of the obstruction of the input and output loops were the long intestine climbing and the angular deformity of the anastomotic opening caused by the operation, we also tried to use the full covered SEMS under endoscopy to correct the angle obstruction of anastomotic loop after gastrectomy. The treatment of anastomotic angular malformation by SEMS under endoscope is a treatment method with small risk, less trauma, quick recovery, and easy to be accepted by patients.

In the study of anastomotic complications, the occurrence time of anastomotic stenosis in upper and lower digestive tracts was 3.83 .46 months and $7.97 \pm 8.6$ months, respectively. The main reason is that the symptoms of upper gastrointestinal tract stenosis are more obvious than those of lower gastrointestinal tract, and the symptoms such as obstruction or abdominal distension after eating may promote the early diagnosis of anastomotic stenosis. Among the 88 patients with anastomotic complications, the postoperative clinical remission or cure rate was $79.5 \%(70 / 88)$. In laboratory examination, the ratio of white blood cells and neutrophils of the patients after the operation did not increase significantly compared with that before the operation, and there was no significant statistical significance.

There are also deficiencies in this study. First, this study is a retrospective observation and analysis. No control group is set for comparative analysis. It is only a summary of the therapeutic effect of endoscopic techniques. Second, the sample size of some types of anastomotic complications was insufficient, and the relevant results were biased. Thirdly, the causes of anastomotic complications were not summarized. Despite relevant defects, there are very few studies on anastomotic complications of large-sample endoscopic treatment at present, and our comprehensive research data can provide reference for endoscopic surgery research.

\section{Conclusions}

In conclusion, for the management of anastomotic complications after digestive tract surgery, the treatment of anastomotic complications under endoscopic support is a minimally invasive, safe, effective, low-risk, quick recovery, and acceptable treatment for patients. At the same time, the long-term 
effect of the treatment of anastomotic complications after digestive tract surgery under the support of endoscopy still needs to be further explored by clinicians.

\section{Abbreviations}

AF: anastomotic fistula; AL: anastomoticleakage; AS: anastomotic stricture; SEMS: self-expandable metal stent; El: endoscopic incision; EBD: balloon dilatation; AO: anastomotic obstruction; OTSC: over-the-scope Clip;

\section{Declarations}

\section{Ethics approval and consent to participate}

This study was approved by the Ethics Committee of Tongji Medical College, Huazhong University of Science and Technology (No. 2018-S377, dated 11 July 2018).All study participants provided written informed consent prior to enrollment.

\section{Consent for publication}

Not applicable.

\section{Availability of data and material}

The authors declare that the data supporting the findings of the present study are available within the manuscript or from the corresponding authors upon reasonable request.

\section{Competing interests}

The authors declare that they have no conflict of interest.

\section{Funding}

All authors confirm that there is no financial support for this article.

\section{Authors' contributions}

Shenghe Deng, Junnan Gu,Yinghao Cao contributed equally to this work. Shenghe Deng, Junnan Gu,Ke Liu contributed to the study design and literature search.Shenghe Deng,Junnan Gu, Yinghao Cao contributed to the literature search and the writing of the manuscript. Ke Wu,Jiliang Wang and Kailin Cai contributed to the ssessment of literature quality,review and revise of the manuscript. Ke Wu and Kailin Cai are the correspondent authors.

\section{Acknowledgments}


The authors sincerely thank the entire staff of our department for offering their assistance with the medical service and manuscript writing processes.

\section{References}

1. Siegel RL, Miller KD, Jemal A. Cancer statistics, 2016. CA Cancer J Clin. 2016 Jan-Feb;66(1):7-30. doi: 10.3322/caac. 21332.

2. Deguchi Y, Fukagawa T, Morita S, Ohashi M, Saka M, Katai H. Identification of risk factors for esophagojejunal anastomotic leakage after gastric surgery. World J Surg. 2012 Jul;36(7):1617-22. doi: 10.1007/s00268-012-1559-3.

3. Rizk NP, Bach PB, Schrag D, Bains MS, Turnbull AD, Karpeh M, Brennan MF, Rusch VW. The impact of complications on outcomes after resection for esophageal and gastroesophageal junction carcinoma. J Am Coll Surg. 2004 Jan;198(1):42-50. doi: 10.1016/j.jamcollsurg.2003.08.007.

4. Patil PK, Patel SG, Mistry RC, Deshpande RK, Desai PB. Cancer of the esophagus: esophagogastric anastomotic leak--a retrospective study of predisposing factors. J Surg Oncol. 1992 Mar;49(3):163-7. doi: 10.1002/jso.2930490307.

5. Cereatti F, Grassia R, Drago A, Conti CB, Donatelli G. Endoscopic management of gastrointestinal leaks and fistulae: What option do we have? World J Gastroenterol. 2020 Aug 7;26(29):4198-4217. doi: 10.3748/wjg.v26.i29.4198.

6. Peifer KJ, Shiels AJ, Azar R, Rivera RE, Eagon JC, Jonnalagadda S. Successful endoscopic management of gastrojejunal anastomotic strictures after Roux-en-Y gastric bypass. Gastrointest Endosc. 2007 Aug;66(2):248-52.doi: 10.1016/j.gie.2006.10.012.

7. Irani S, Kozarek RA. Techniques and principles of endoscopic treatment of benign gastrointestinal strictures. Curr Opin Gastroenterol. 2015 Sep;31(5):339-50.doi: 10.1097/MOG.0000000000000200.

8. World Medical Association. World Medical Association Declaration of Helsinki: ethical principles for medical research involving human subjects. JAMA. 2013 Nov 27;310(20):2191-4.doi: 10.1001/jama.2013.281053.

9. Song YN, Qi Y, Zhang CY, Sheng YL, Wu K, Zhu SL, Han L, Shan TT, Ye GC, Zhang QY, Chen YL, Chen JW, Liu YF, Gao LB, Yang Y, He ZF, Zhu DY, Liu DL, Wen FB, Zheng TL, Li JL, Zhao S. A new technology for reducing anastomotic fistula in the neck after esophageal cancer surgery. $J$ Thorac Dis. 2019 Jul;11(7):3084-3092.doi: 10.21037/jtd.2019.07.28.

10. Truong S, Willis S, Schumpelick V. Endoscopic therapy of benign anastomotic strictures of the colorectum by electroincision and balloon dilatation. Endoscopy. 1997 Nov;29(9):845-9.doi: 10.1055/s-2007-1004319.

11. Low DE, Alderson D, Cecconello I, Chang AC, Darling GE, D'Journo XB, Griffin SM, Hölscher AH, Hofstetter WL, Jobe BA, Kitagawa Y, Kucharczuk JC, Law SY, Lerut TE, Maynard N, Pera M, Peters JH, Pramesh CS, Reynolds JV, Smithers BM, van Lanschot JJ. International Consensus on Standardization of Data Collection for Complications Associated With Esophagectomy: 
Esophagectomy Complications Consensus Group (ECCG). Ann Surg. 2015 Aug;262(2):286-94. doi: 10.1097/SLA.0000000000001098.

12. van den Berg MW, Kerbert AC, van Soest EJ, Schwartz MP, Bakker CM, Gilissen LP, van Hooft JE. Safety and efficacy of a fully covered large-diameter self-expanding metal stent for the treatment of upper gastrointestinal perforations, anastomotic leaks, and fistula. Dis Esophagus. 2016 Aug;29(6):572-9.doi: 10.1111/dote.12363.

13. Yimcharoen P, Heneghan HM, Tariq N, Brethauer SA, Kroh M, Chand B. Endoscopic stent management of leaks and anastomotic strictures after foregut surgery. Surg Obes Relat Dis. 2011 Sep-Oct;7(5):628-36. doi: 10.1016/j.soard.2011.03.017.

14. Eubanks S, Edwards CA, Fearing NM, Ramaswamy A, de la Torre RA, Thaler KJ, Miedema BW, Scott JS. Use of endoscopic stents to treat anastomotic complications after bariatric surgery. J Am Coll Surg. 2008 May;206(5):935-8; discussion 938-9. doi: 10.1016/j.jamcollsurg.2008.02.016.

15. Biraima M, Adamina M, Jost R, Breitenstein S, Soll C. Long-term results of endoscopic balloon dilation for treatment of colorectal anastomotic stenosis. Surg Endosc. 2016 Oct;30(10):4432-7.doi: 10.1007/s00464-016-4762-8.

16. Araujo SE, Costa AF. Efficacy and safety of endoscopic balloon dilation of benign anastomotic strictures after oncologic anterior rectal resection: report on 24 cases. Surg Laparosc Endosc Percutan Tech. 2008 Dec;18(6):565-8. doi: 10.1097/SLE.0b013e31818754f4.

17. Fregonese D, Di Falco G, Di Toma F. Balloon dilatation of anastomotic intestinal stenoses: long-term results. Endoscopy. 1990 Nov;22(6):249-53. doi: 10.1055/s-2007-1012863.

18. Tan Y, Lv L, Duan T, Zhou J, Liu D. Endoscopic incision and selective cutting for the treatment of refractory colorectal anastomotic strictures. Int J Colorectal Dis. 2016 May;31(5):1063-1064.doi: 10.1007/s00384-015-2385-5.

19. Osera S, Ikematsu H, Odagaki T, Oono Y, Yano T, Kobayashi A, Ito M, Saito N, Kaneko K. Efficacy and safety of endoscopic radial incision and cutting for benign severe anastomotic stricture after surgery for lower rectal cancer (with video). Gastrointest Endosc. 2015 Mar;81(3):770-3. doi: 10.1016/j.gie.2014.11.011.

20. Deng S, Cao Y, Gu J, Wu K, Li J, Tao K, Wang G, Wang J, Cai K. Endoscopic diagnosis and treatment of complete anastomosis stenosis after colorectal resection without protective ileostomy: report of two cases and literature review. J Int Med Res. 2020 Apr;48(4):300060520914833. doi: $10.1177 / 0300060520914833$.

21. Davis B, Rivadeneira DE. Complications of colorectal anastomoses: leaks, strictures, and bleeding. Surg Clin North Am. 2013 Feb;93(1):61-87. doi: 10.1016/j.suc.2012.09.014.

22. Hirst NA, Tiernan JP, Millner PA, Jayne DG. Systematic review of methods to predict and detect anastomotic leakage in colorectal surgery. Colorectal Dis. 2014 Feb;16(2):95-109.doi: 10.1111/codi.12411.

23. Akashi Y, Hiki N, Nunobe S, Jiang X, Yamaguchi T. Safe management of anastomotic leakage after gastric cancer surgery with enteral nutrition via a nasointestinal tube. Langenbecks Arch Surg. 2012 
Jun;397(5):737-44.

24. Junemann-Ramirez M, Awan MY, Khan ZM, Rahamim JS. Anastomotic leakage postesophagogastrectomy for esophageal carcinoma: retrospective analysis of predictive factors, management and influence on longterm survival in a high volume centre. Eur $\mathrm{J}$ Cardiothorac Surg. 2005 Jan;27(1):3-7. doi: 10.1007/s00423-012-0935-7.

25. Rustagi T, McCarty TR, Aslanian HR. Endoscopic Treatment of Gastrointestinal Perforations, Leaks, and Fistulae. J Clin Gastroenterol. 2015 Nov-Dec;49(10):804-9. doi:

10.1097/MCG.0000000000000409.

26. Chang J, Sharma G, Boules M, Brethauer S, Rodriguez J, Kroh MD. Endoscopic stents in the management of anastomotic complications after foregut surgery: new applications and techniques. Surg Obes Relat Dis. 2016 Aug;12(7):1373-1381. doi: 10.1016/j.soard.2016.02.041.

27. Law JK, Stoita A, Wever W, Gleeson FC, Dries AM, Blackford A, Kiswani V, Shin EJ, Khashab MA, Canto MI, Singh VK, Lennon AM. Endoscopic ultrasound-guided fine needle aspiration improves the pre-operative diagnostic yield of solid-pseudopapillary neoplasm of the pancreas: an international multicenter case series (with video). Surg Endosc. 2014 Sep;28(9):2592-8. doi: 10.1007/s00464-0143508-8.

28. Mussetto A, Arena R, Buzzi A, Fuccio L, Dari S, Brancaccio ML, Triossi O. Long-term efficacy of vacuum-assisted therapy (Endo-SPONGE $®$ ) in large anastomotic leakages following anterior rectal resection. Ann Gastroenterol. 2017;30(6):649-653. doi: 10.20524/aog.2017.0194.

\section{Figures}


A

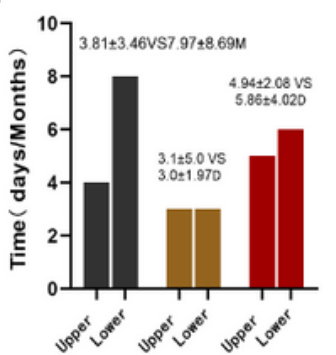

C

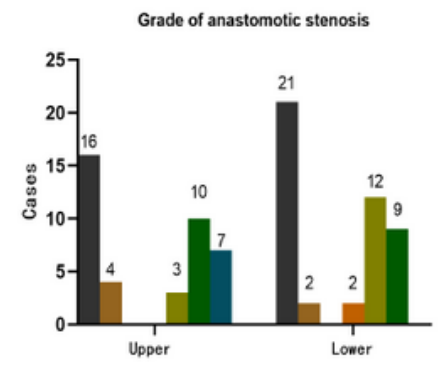

E

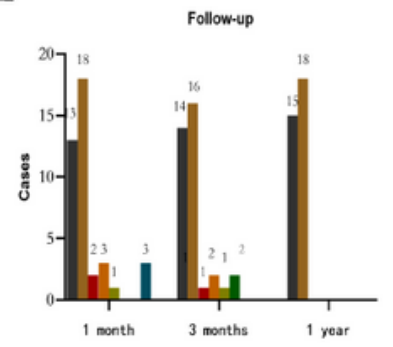

G

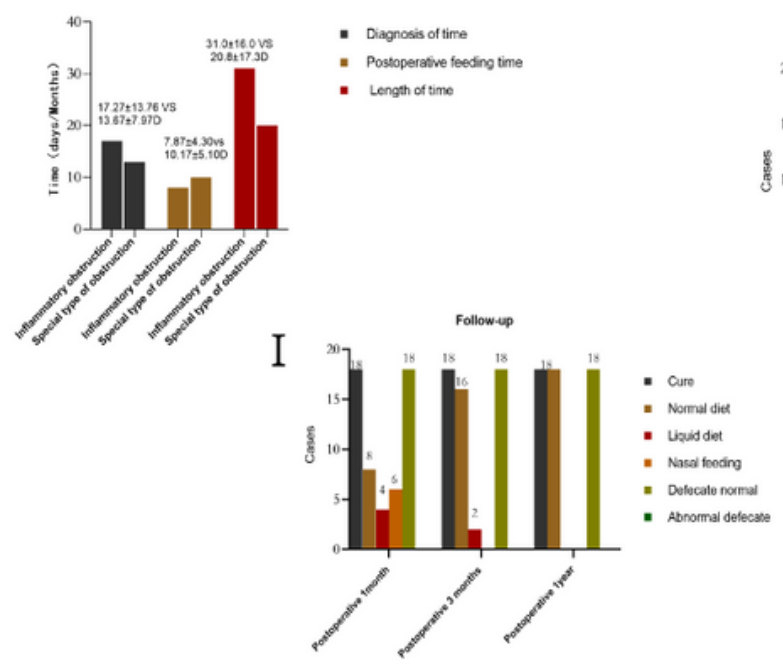

B

- Diagnosis of time

- Postoperative feeding time

- length of stay

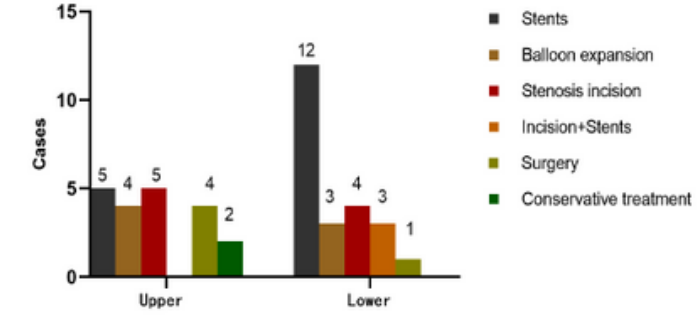

D

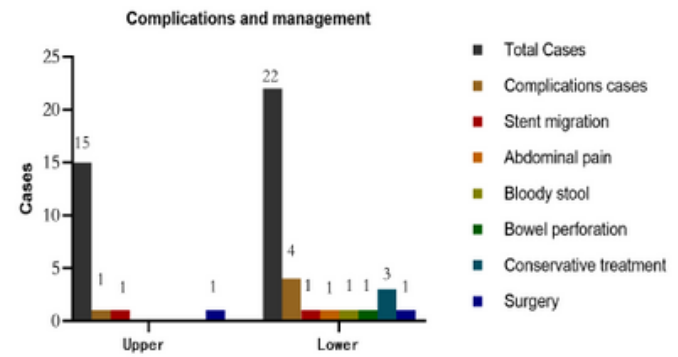

F

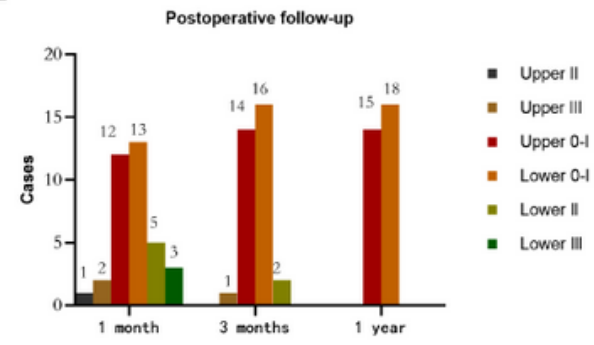

$\mathrm{H}$

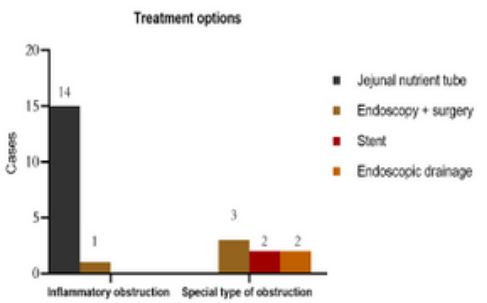

\section{Figure 1}

Anastomotic stenosis, anastomotic obstruction clinicopathological features and endoscopic treatment. A: General situation of patients with anastomotic stenosis; B: Endoscopic treatment for patients with anastomotic stenosis; $\mathrm{C}$ stenosis classification of patients with anastomotic stenosis; D: Complications and management of endoscopic treatment of anastomotic stenosis; E: Recovery of patients with anastomotic stenosis; F:One-year follow-up of patients with anastomotic stenosis.G: General situation of 
patients with anastomotic obstruction. $\mathrm{H}$ : Endoscopic treatment for patients with anastomotic obstruction; I:One-year follow-up of patients with anastomotic obstruction.

A

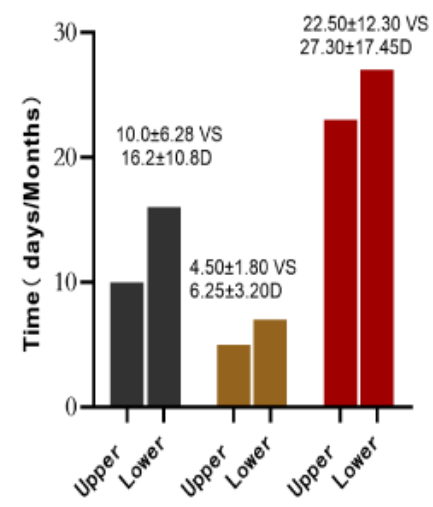

C

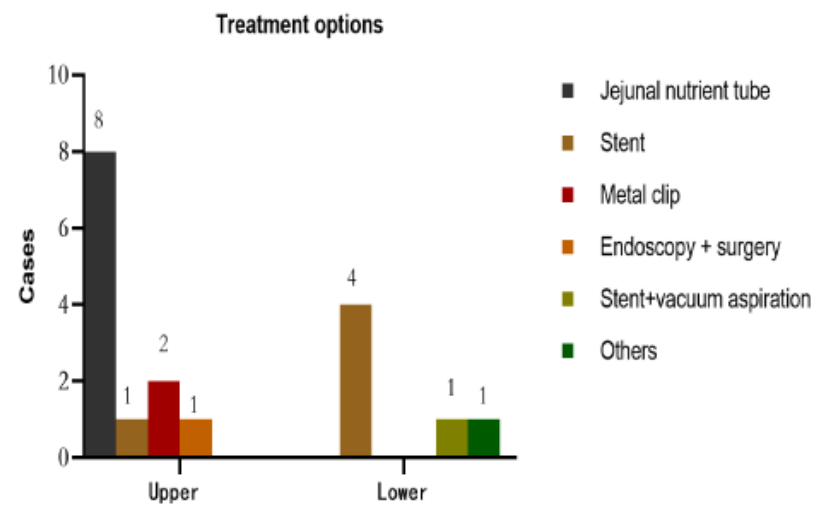

$\mathrm{E}$

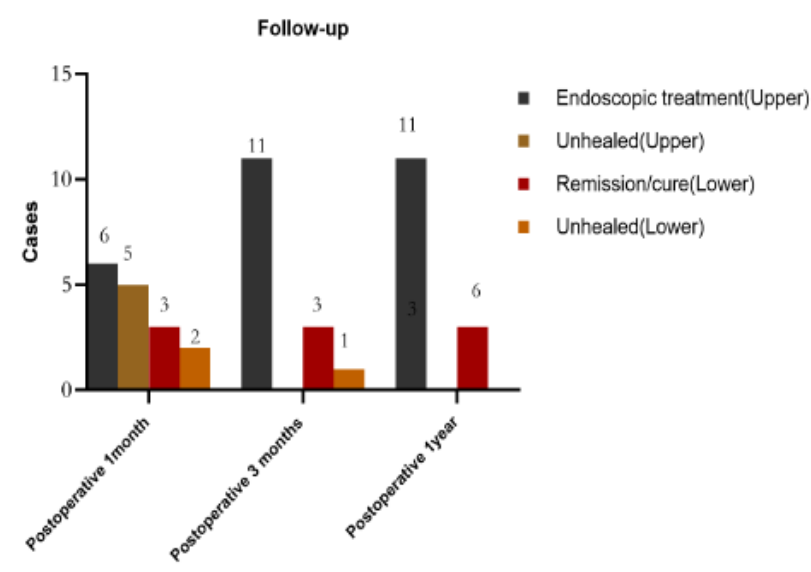

B

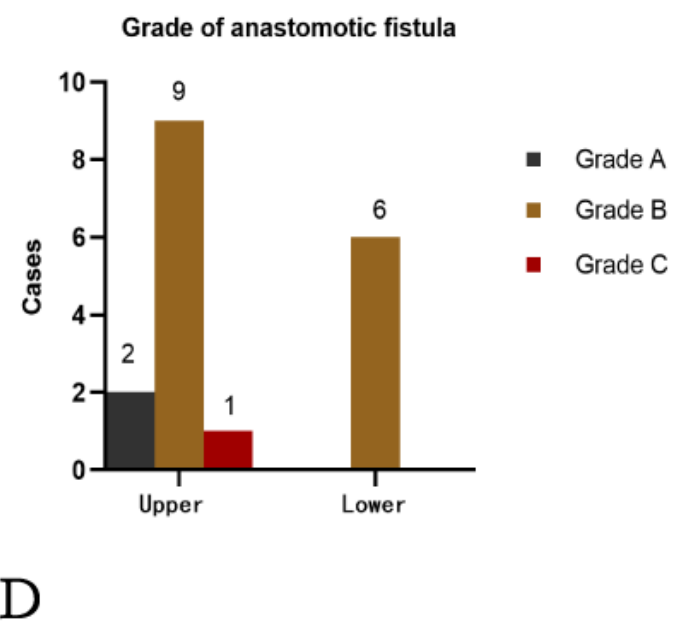

$\mathrm{F}$

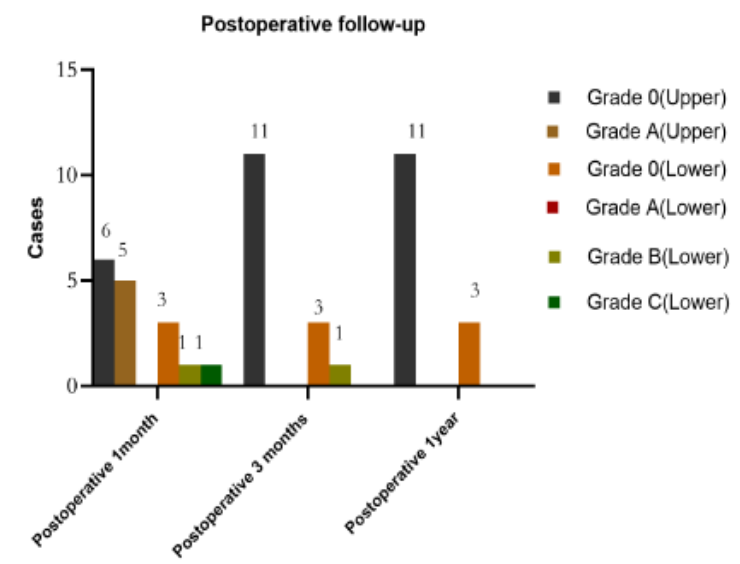

Figure 2

Clinicopathological features and endoscopic treatment of anastomotic fistula. A: General information of anastomotic fistula patients; B: Classification of anastomotic fistula; C: Endoscopic treatment for patients with anastomotic fistula; D: Complications and treatment of anastomotic fistula endoscopic 
treatment; E: Remission in patients with anastomotic fistula; F: Anastomotic fistula was followed up for 1 year.

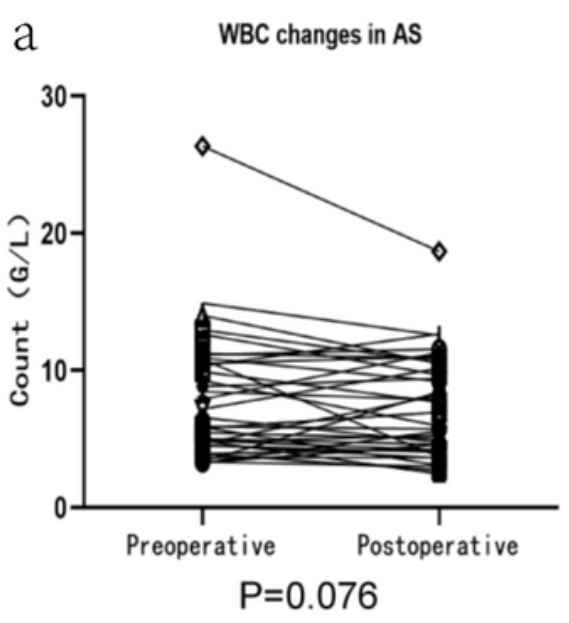

C

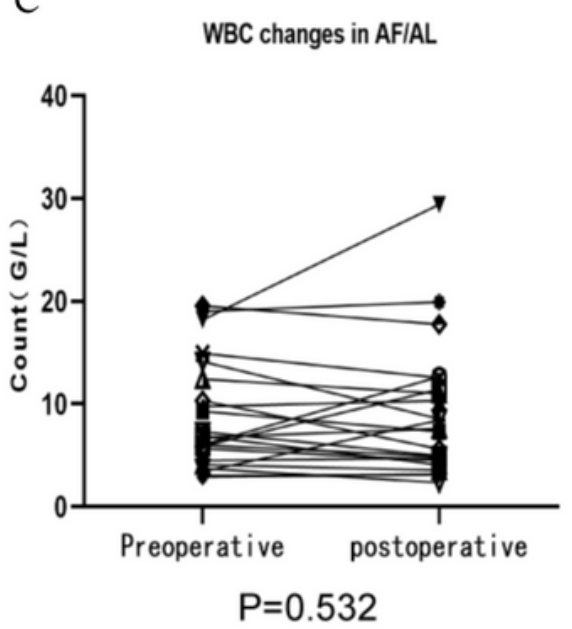

e

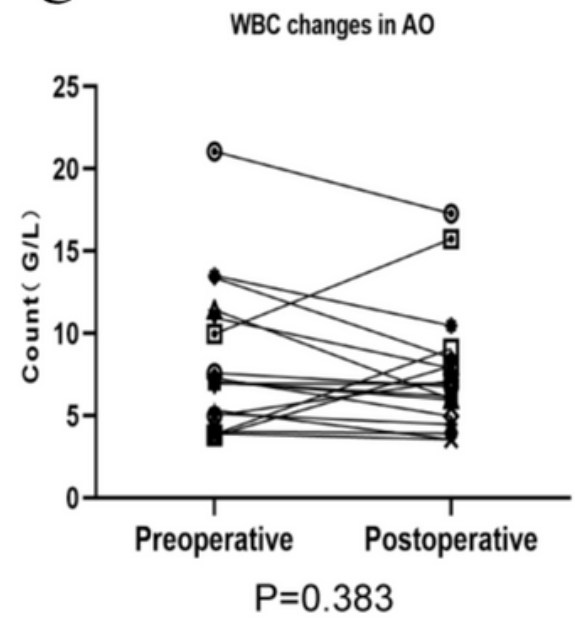

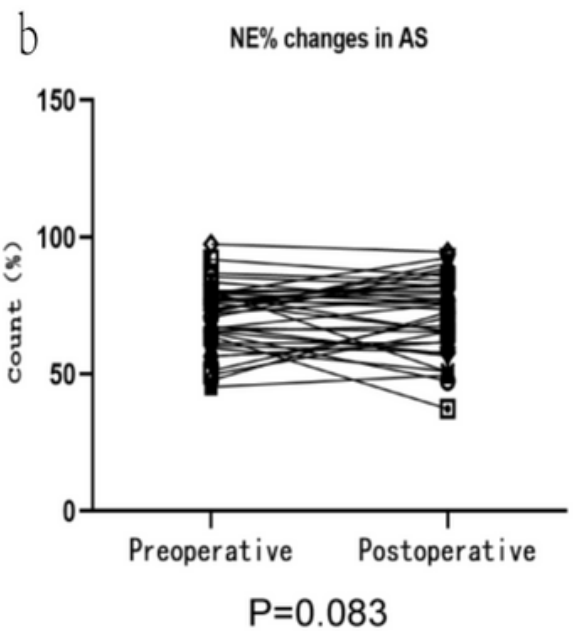

d
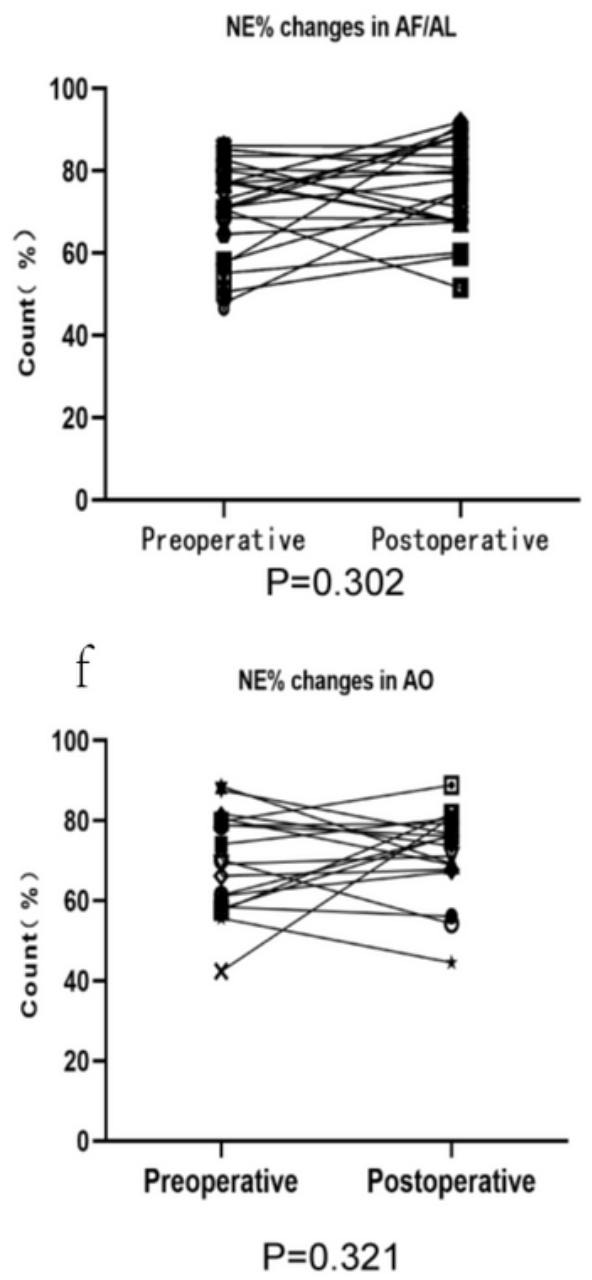

\section{Figure 3}

(A): Changes in WBC and NE\% before and after endoscopic treatment for anastomotic stenosis; (B): Changes in WBC and NE\% before and after endoscopic treatment of anastomotic fistula; (C): Changes in WBC and NE\% before and after endoscopic treatment for anastomotic obstruction. 


\section{Supplementary Files}

This is a list of supplementary files associated with this preprint. Click to download.

- Tables1.doc

- Tables2.doc

- Tables3.doc

- Tables4.doc

- Tables5.doc

- Tables6.doc

- Tables7.doc 